\title{
Changes in mitochondrial function and mitochondria associated protein expression in response to 2-weeks of high intensity interval training
}

\author{
Grace Vincent ${ }^{1,2 \ddagger}$, Séverine Lamon ${ }^{1 \neq}$, Nicholas Gant ${ }^{2}$, Peter J. Vincent ${ }^{3}$, Julia R. MacDonald ${ }^{4}$, \\ James F. Markworth ${ }^{5}$, Johann A. Edge ${ }^{2+}$ and Anthony J. R. Hickey ${ }^{4 *}$ \\ ${ }^{1}$ Centre for Physical Activity and Nutrition Research, School of Exercise and Nutrition Sciences, Deakin University, Melbourne, VIC, Australia \\ ${ }^{2}$ Department of Sport and Exercise Science, The University of Auckland, Auckland, New Zealand \\ ${ }^{3}$ Department of General Practice and Primary Healthcare, Auckland School of Medicine, The University of Auckland, Auckland, New Zealand \\ ${ }^{4}$ Applied Surgery and Metabolism Laboratory, School of Biological Sciences, The University of Auckland, Auckland, New Zealand \\ ${ }^{5}$ Liggins Institute, The University of Auckland, Auckland, New Zealand
}

\section{Edited by:}

Julian Stelzer, Case Western

Reserve University, USA

Reviewed by:

Robert W. Wiseman, Michigan State

University, USA

Jessica M. Berthiaume, Case

Western Reserve University, USA

*Correspondence:

Anthony J. R. Hickey, Applied

Surgery and Metabolism Laboratory,

School of Biological Sciences, The

University of Auckland, Thomas

Building, Auckland 1010, New

Zealand

e-mail:a.hickey@auckland.ac.nz

${ }^{\dagger}$ Deceased.

${ }^{\ddagger}$ Co-first authors.
Purpose: High-intensity short-duration interval training (HIT) stimulates functional and metabolic adaptation in skeletal muscle, but the influence of HIT on mitochondrial function remains poorly studied in humans. Mitochondrial metabolism as well as mitochondrial-associated protein expression were tested in untrained participants performing HIT over a 2-week period.

Methods: Eight males performed a single-leg cycling protocol $(12 \times 1 \mathrm{~min}$ intervals at $120 \%$ peak power output, 90 s recovery, 4 days/week). Muscle biopsies (vastus lateralis) were taken pre- and post-HIT. Mitochondrial respiration in permeabilized fibers, citrate synthase (CS) activity and protein expression of peroxisome proliferator-activated receptor gamma coactivator (PGC-1 $\alpha$ ) and respiratory complex components were measured.

Results: HIT training improved peak power and time to fatigue. Increases in absolute oxidative phosphorylation (OXPHOS) capacities and CS activity were observed, but not in the ratio of $\mathrm{CCO}$ to the electron transport system (CCO/ETS), the respiratory control ratios (RCR-1 and RCR-2) or mitochondrial-associated protein expression. Specific increases in OXPHOS flux were not apparent after normalization to CS, indicating that gross changes mainly resulted from increased mitochondrial mass.

Conclusion: Over only 2 weeks HIT significantly increased mitochondrial function in skeletal muscle independently of detectable changes in mitochondrial-associated and mitogenic protein expression.

Keywords: HIT, mitochondria, oxidative phosphorylation, PGC-1 $\alpha$, skeletal muscle

\section{INTRODUCTION}

High-intensity, short-duration interval training (HIT) rapidly stimulates metabolic adaptations in skeletal muscle and improves aerobic capacity (Parra et al., 2000; Burgomaster et al., 2005, 2006, 2007, 2008; Gibala et al., 2006a, 2012; Gibala and McGee, 2008; Babraj et al., 2009). HIT is more effective than moderateintensity continuous training for increasing aerobic power and managing risk factors related to the metabolic syndrome (Tjonna et al., 2008). So far, the majority of HIT research has used highly demanding training protocols typically including repetitive "all out" maximal sprinting efforts of $\sim 30 \mathrm{~s}$ (Burgomaster et al., 2005; Gibala et al., 2006a). However, intense anaerobic exercise requires considerable participant motivation, which likely impacts its general prescription. Recently, more practical HIT models have been introduced, involving bouts of extended duration at a lower intensity interspersed with appropriate recovery periods (Little et al., 2011; Jacobs et al., 2013b). This modified form of HIT improves aerobic capacity and results in concurrent changes in mitochondrial associated mRNA and protein levels as well as in mitochondrial enzyme activity, indicating an enhanced oxidative potential (Hood et al., 2011; Little et al., 2011). Improvements in markers of metabolic control and arterial compliance are comparable to traditional endurance exercise training (Burgomaster et al., 2008; Rakobowchuk et al., 2008) and HIT is perceived to be more enjoyable (Bartlett et al., 2011). Therefore, HIT likely represents a time-efficient strategy to enhance whole body physiological function and prevent metabolic disease. However, few studies have explored the metabolic adaptations through which these improvements in exercise performance occur (Jacobs et al., 2013b).

Traditional endurance exercise protocols promote mitochondrial content (Holloszy, 1967; Bruce et al., 2004) and mitochondrial biogenesis (Wright et al., 2007a). Molecular signals such as $\mathrm{Ca}^{+2}, 5^{\prime}$ adenosine monophosphate (AMP) and reactive oxygen 
species (ROS) increase post-exercise leading to the initial activation of mitochondrial biogenesis (McConell et al., 1997; Baar et al., 2003; Irrcher et al., 2009). The underlying exercise-induced mechanisms following cumulative exercise sessions have been well documented (Wright et al., 2007a), resulting in enhanced levels of peroxisome proliferator-activated receptor gamma coactivator (PGC-1 $\alpha$ ) gene expression. PCG-1 $\alpha$, a key regulator of mitochondrial biogenesis and function that positively regulates the mitochondrial network (Wu et al., 1999; Finck and Kelly, 2006; Handschin and Spiegelman, 2006), is upregulated in human skeletal muscle following acute endurance exercise (Cartoni et al., 2005; Russell et al., 2005) and low-volume HIT (Gibala et al., 2009; Little et al., 2010).

Short duration HIT has been shown to improve aerobic capacity (Burgomaster et al., 2005) and elevate mitochondrial enzyme activities (Gibala et al., 2006c; Hood et al., 2011; Little et al., 2011). Enzyme activity assays and mitochondrial-associated gene and protein expression provide static, surrogate measures of mitochondrial content and oxidative capacities. However, changes in these markers do not always reflect the collective function or the complexity of mitochondrial function (Larsen et al., 2012; Jacobs et al., 2013a). Indeed, they do not provide a measure of oxygen flux capacities (e.g., of the oxidative phosphorylation system (OXPHOS), and maximal uncoupled respiration in permeabilized fibers) or phosphorylation coupling efficiencies (Picard et al., 2011). In addition, alterations in mitochondrial function can occur independently from changes in mitochondrial associated protein expression (Viganò et al., 2008; Jacobs et al., 2013a). The permeabilized fiber method allows measurement of mitochondrial respiration in situ from as little as $2.5 \mathrm{mg}$ of muscle (Pesta and Gnaiger, 2012), and provides a reference to the maximal oxidative power in vivo (Boushel et al., 2011), as well as an estimate of energy transfer efficiency through the degree of mitochondrial respiratory control. Furthermore, in situ analysis minimizes mitochondrial disruption that inevitably occurs during the isolation process (Daussin et al., 2008; Picard et al., 2011).

To our knowledge, only one previous study investigated mitochondrial function following a short period of HIT (Jacobs et al., 2013b). In addition, discrepancies in mitochondrial function and protein expression are not without precedent in the literature (Viganò et al., 2008; Jacobs et al., 2013a). However, mitochondrial function and mitochondrial associated protein expression have never been investigated in parallel following a HIT regime. Therefore, the aim of the present study was to investigate the changes in respiratory fluxes that underlie exercise performance responses to HIT using the permeabilized fiber method, as well as the expression levels of proteins associated with mitochondrial function. We hypothesized that 8 sessions of HIT over 2 weeks would increase markers of mitochondrial function and mitochondrial content within skeletal muscle.

\section{MATERIALS AND METHODS PARTICIPANTS}

Eight male, moderately active participants (Mean \pm SD, age $22 \pm 2$ years, body mass $81 \pm 6 \mathrm{~kg}$, height $1.82 \pm 0.1 \mathrm{~m}$ ) volunteered to participate in the study. Participants were screened for contraindications to exercise and gave written consent before participating. The study design and experimental procedures were approved by The University of Auckland Human Participants Ethics Committee (Ref. 2009/397).

\section{FAMILIARIZATION AND BASELINE EXERCISE TESTING}

This study was originally part of a larger trial aimed at looking at cross-education between limbs (Howatson et al., 2013; Magnus et al., 2013; Pearce et al., 2013). The foot of the contralateral leg was firmly strapped to a platform that replaced the crank arm on the non-exercising side. As we did not observe any exerciseinduced change in any of the measured parameters, we decided to focus on the exercising leg only. Therefore, one-legged cycling was selected as the training modality. A preliminary session was conducted to familiarize participants with the one-legged cycling exercise mode. This comprised one $10 \mathrm{~min}$ bout of cycling at an intensity of $100 \mathrm{~W}$ for each leg, performed unilaterally. Forty eight hours post-familiarization participants performed two onelegged cycling graded exercise tests to volitional fatigue on an electromagnetically braked cycle ergometer (Schiller CH-6340, BAAR, Switzerland) to determine baseline peak power, time to fatigue, and $\mathrm{VO}_{2}$ peak.

The $\mathrm{VO}_{2}$ peak test commenced at a workload of $65 \mathrm{~W}$ with step-wise increments of $15 \mathrm{~W}$ every $2 \mathrm{~min}$ until cadence fell below $60 \mathrm{rpm}$. An open-circuit gas analysis system (Moxus modular oxygen uptake system, AEI technologies, Pittsburgh, PA, USA) was used to analyze expired air and determine peak oxygen consumption. The gas analysis system was calibrated prior to each test. Five days after the preliminary session, bilateral resting muscle biopsy samples were collected from vastus lateralis.

\section{TRAINING PROTOCOL}

Each participant was assigned a leg for HIT that was randomized by limb dominance in a counterbalanced fashion. The trained leg performed $12 \times 60 \mathrm{~s}$ intervals at $120 \%$ of peak power (as determined by the preliminary $\mathrm{VO}_{2}$ peak test) with a $90 \mathrm{~s}$ rest between intervals on the cycle ergometer. Sessions were performed 4 days per week for 2 weeks ( 8 sessions in total). A researcher measured recovery intervals and provided encouragement to facilitate maximal effort for each high-intensity interval. As in the exercise capacity tests, participants were required to remain seated during the training period. Participants refrained from participating in additional strenuous aerobic exercise or strength training outside of the prescribed training sessions during the study period. All participants completed all training sessions.

\section{POST-INTERVENTION TESTS}

Approximately $48 \mathrm{~h}$ after completion of the last training session, a muscle biopsy sample was taken from each leg following an overnight fast. A further $48 \mathrm{~h}$ elapsed before participants performed two unilateral one-legged graded exercise tests to determine changes in peak power, time to fatigue, and $\mathrm{VO}_{2}$ peak.

\section{SKELETAL MUSCLE SAMPLING AND FIBER PREPARATION}

Local anesthetic (2\% Xylocaine) was injected into the skin overlying the vastus lateralis and a small incision made to the skin and underlying tissue. A $5 \mathrm{~mm}$ Bergstrom needle was then inserted into the belly of the vastus lateralis muscle. Manual suction 
was applied and the biopsy sample excised. Extracted tissue was then divided into two $\sim 50 \mathrm{mg}$ samples, and a sample for mitochondrial respiration analysis was washed with saline solution and placed into an $1.7 \mathrm{ml}$ micro-centrifuge tube containing ice-cold high energy relaxing solution (BIOPS, from here on in $\mathrm{mmol} \cdot \mathrm{L}^{-1}$ unless stated $10 \mathrm{Ca}$-EGTA buffer, 0.1 free calcium, 20 imidazole, 20 taurine, $50 \mathrm{~K}$-MES (potassium 2- $(\mathrm{N}$ morpholino)ethanesulfonic acid), 0.5 dithiothreitol, $6.56 \mathrm{MgCl}_{2}$, 5.77 ATP, 15 phosphocreatine, at $\mathrm{pH}$ 7.1. The remaining sample was frozen immediately in liquid nitrogen and stored in $\mathrm{a}-80^{\circ} \mathrm{C}$ freezer for later analysis.

BIOPS muscle stored was teased into small fiber bundles within $2 \mathrm{~h}$ of collection, as previously described (Pesta and Gnaiger, 2012). Muscle fiber bundles were then placed into $1 \mathrm{ml}$ ice-cold BIOPS and freshly prepared saponin $(50 \mu \mathrm{g})$ was added to permeabilize the plasma membrane. Fibers were then gently shaken in culture plates for $30 \mathrm{~min}$ at $4^{\circ} \mathrm{C}$. Fibers were rinsed 3 times for $10 \mathrm{~min}$ in $1 \mathrm{ml}$ ice-cold incubation MiRO5 assay medium (0.5 EGTA, $3 \mathrm{MgCl}_{2}, 60 \mathrm{~K}$-lactobionate, 20 taurine, $10 \mathrm{KH}_{2} \mathrm{PO}_{4}, 110$ sucrose, and $1 \mathrm{mg} \cdot \mathrm{ml}^{-1} \mathrm{BSA}$ in $20 \mathrm{HEPES}$, $\mathrm{pH} 7.1$ at $30^{\circ} \mathrm{C}$ ) to remove the saponin, cytosol, and adenine nucleotides. Fiber bundles were blotted dry using filter paper and $5 \mathrm{mg}$ of skeletal muscle tissue weighed for respiration assays.

\section{MITOCHONDRIAL RESPIRATION}

Mitochondrial respiration in permeabilized muscle fibers was analyzed using two OROBOROS ${ }^{\circledR}$ O2K oxygraphs (Anton Paar, Graz, Austria) with chambers adjusted to $2 \mathrm{ml}$ at $30^{\circ} \mathrm{C}$. Respiration was measured as weight-specific oxygen flux $\left(\mathrm{pmolO}_{2} \cdot \mathrm{s}^{-1} \cdot \mathrm{mg}^{-1}\right.$ ) wet weight, calculated as the time derivative of oxygen concentration using the DatLab 4 Analysis Software, OROBOROS $^{\circledR}$ (Oroboros Instruments, Innsbruck, Austria).

The assay protocol consisted of the sequential titration of various substrates, inhibitors, and uncoupling agents of the respiratory chain to the skeletal muscle in MiRO5 containing catalase $\left(1558 \mathrm{U} \cdot \mathrm{ml}^{-1}\right)$. Fibers were super-saturated with oxygen by injecting oxygen into the header space above samples after calibration and levels maintained between 280 and $450 \mathrm{nmol} \cdot \mathrm{ml}^{-1}$. All assays were performed in duplicate.

We chose a substrate/inhibitor titration protocol targeting fluxes through aerobic glycolytic routes. Basal oxidation was first measured, then $10 \mathrm{mM}$ glutamate and $2 \mathrm{mM}$ malate were added and the LEAK state measured (GM leak). The addition of excess 1.25 mM ADP tested Complex I (CI) dependent oxidative phosphorylation with GM (GM-ADP), and the addition of cytochrome- $c(10 \mu \mathrm{M})$ tested the functional integrity of mitochondrial outer membranes. The additional effect of pyruvate $(10 \mathrm{mM})$ was used to maximize CI mediated flux (OXPHOS-CI) followed by the Complex II (CII) substrate succinate $(10 \mathrm{mM})$ for combined Complex I and Complex II flux (OXPHOS-CI, $\mathrm{CII}$ ), and this combination of substrates aimed to maximize mitochondrial $\mathrm{O}_{2}$ flux capacities.

Inhibition of the phosphorylation system by addition of atractyloside $(0.25 \mathrm{mM})$ resulted in a transition to State 4 respiration (CI, CII Leak) by blocking the adenine nucleotide translocase (ANT). In excess ADP, like GDP inhibits uncoupling protein conductance (UCPs). As atractyloside also inhibits proton leak through ANT, any residual Leak respiration results from proton flux through the inner mitochondrial membrane.

The electron transport system (ETS) was then uncoupled from the inhibited phosphorylation system by repeated titrations of $1 \mu \mathrm{L}$ of carbonyl cyanide $\mathrm{p}$-(trifluoromethoxy) phenyl-hydrazone (FCCP, $0.5 \mu \mathrm{M}$ ). Under these conditions this state represents the maximal rate of the ETS (ETS CI, CII). Complex I (ETS CII) and then Complex II and III activity were selectively and sequentially inhibited by the addition of rotenone $(1 \mu \mathrm{M}), 15 \mathrm{mM}$ malonate and antimycin- $a(1 \mu \mathrm{M})$, which act to block the flow of electrons from Complexes I, II, and III respectively. Finally, the activity of CCO was measured by addition of the electron donor couple $\mathrm{N}, \mathrm{N}, \mathrm{N}^{\prime}, \mathrm{N}^{\prime}$-tetramethyl-p-phenylendiamine (TMPD, $0.5 \mathrm{mM}$ ) and ascorbate $(2 \mathrm{mM})$. Results were then corrected for background chemical $\mathrm{O}_{2}$ consumption and also normalized to citrate synthase (CS) activity that were measured in matched samples. Control ratios were determined as follows; The RCR-1 = the ratio of oxidative phosphorylation (OXPHOS) derived oxygen flux relative to leak respiration flux supported by complex I (CI) substrates. RCR-2 = the ratio of OXPHOS and leak respiration fluxes supported by CI and Complex II. \% CI/ETS = percentage contribution of CI to the chemically uncoupled ETS oxygen flux. $\mathrm{CCO} / \mathrm{ETS}=$ the ratio of cytochrome $c$ oxidase (CCO) relative to the ETS (CCO/ETS) provided a measure of the flux through CCO relative to the ETS. A representative trace of the respiration substrate, inhibitor and uncoupler titration protocol is shown in Figure 1.

\section{CITRATE SYNTHASE ASSAY}

Stored tissue was equilibrated with 1:40 volumes (w:v) ice-cold homogenization buffer (25 Tris- $\mathrm{HCl} \mathrm{pH} 7.8,1$ EDTA, $2 \mathrm{MgCl}_{2}$, $50 \mathrm{KCl}, 0.5 \% \mathrm{v} / \mathrm{v}$ Triton X-100). Samples were then homogenized and citrate synthase (CS) activity determined using a plate reader (Molecular Devices Spectramax 340, Sunnyvale, USA) and $5 \mu 1$ of sample supernatant was added to an assay media containing Tris- $\mathrm{HCl}$ (50 mM, pH 8.0), acetyl coenzyme A (0.1 mM), DTNB $(0.2 \mathrm{mM})$. Reactions started by addition of $5 \mathrm{mM}$ oxaloacetate (Sigma, St. Louis, USA), and followed the formation of mercaptide ions at $412 \mathrm{~nm}$. CS activity (units per wet weight of tissue) was determined relative to CS standard (Sigma, St. Louis, USA) from porcine heart. CS data were used as a proxy of mitochondrial mass and used to normalize flux rates in order to estimate mitochondrial specific fluxes.

\section{PROTEIN EXTRACTION AND IMMUNOBLOTTING}

Frozen tissue (20-30 mg) was mechanically disrupted in $15 \mu \mathrm{L}$ ice cold lysis buffer/mg (in mM, 20 Tris-HCL (pH 7.4), 137 $\mathrm{NaCl}, 1 \%$ NP-40, 10\% glycerol, 1 EDTA, with Halt Protease, and Phosphatase Inhibitor Cocktail (Thermo Scientific Pierce) with $2.8 \mathrm{~mm}$ ceramic beads using the Omni Bead Ruptor Homogenizer bead mill (Omni International) at $5.65 \mathrm{~m} \cdot \mathrm{s}^{-1}$, $2 \times 30$ s. Homogenates were then rotated for $1 \mathrm{~h}$ at $4^{\circ} \mathrm{C}$ then centrifuged at $13,000 \mathrm{~g}$ for $15 \mathrm{~min}$ at $4^{\circ} \mathrm{C}$ and supernatants stored at $-80^{\circ} \mathrm{C}$. Protein contents were determined by the bicinchoninic acid method (Thermo Scientific Pierce). Samples were diluted to $20 \mu \mathrm{g}$ protein in $4 \times$ Laemmli loading buffer, 


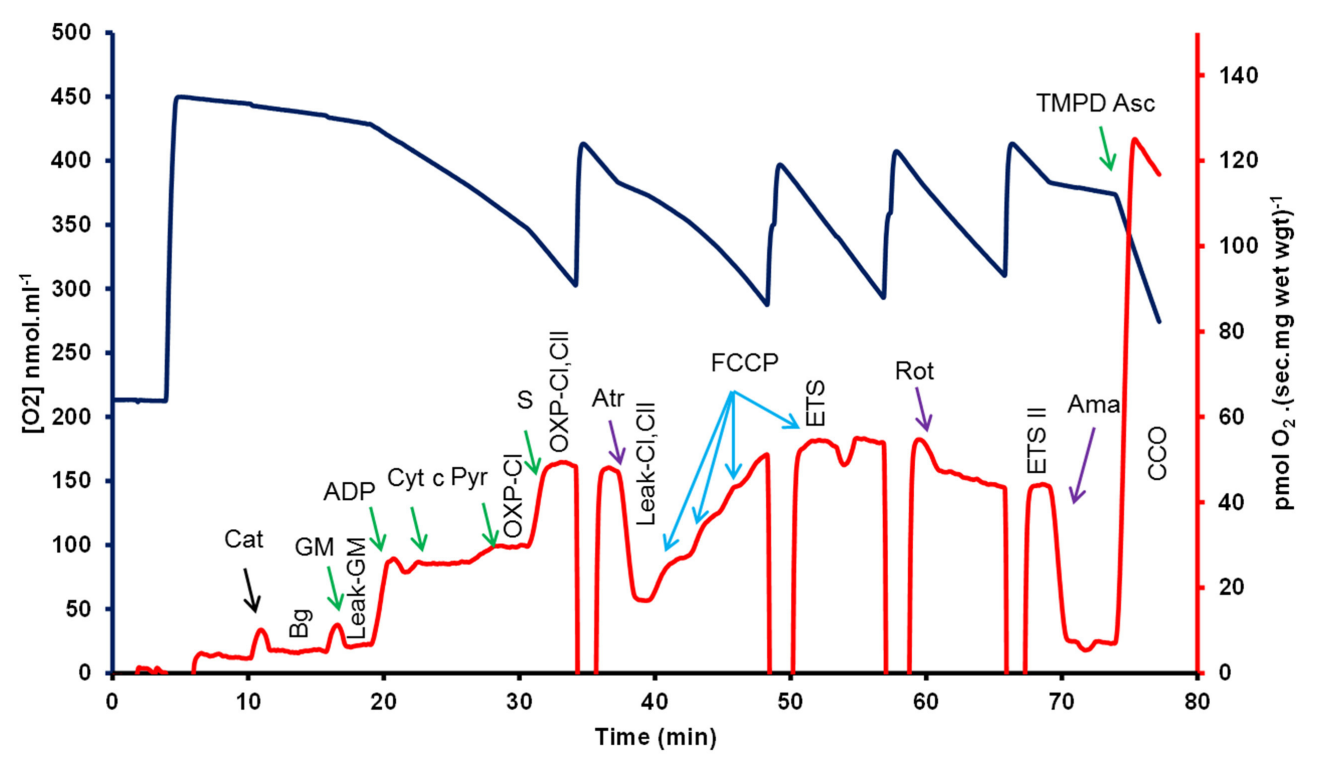

FIGURE 1 | A representative trace of the respiration substrate inhibitor uncoupler titration protocol used in this study to test saponin permeabilized vastus lateralis muscle (pre-HIT). The change in oxygen concentration (blue line) and the time derivative per unit mass (red line and axis) provided the flux of permeabilized vastus lateralis fibers over time following the addition of mitochondrial substrates (green), poisons (purple) and the uncoupling agent FCCP, carbonyl cyanide p-(trifluoromethoxy) phenyl-hydrazone (FCCP, blue). GM-Leak — glutamate and malate, ADP addition initiates oxidative phosphorylation (OXPHOS) with GM. Cyt-c-addition of cytochrome $c$ tested mitochondrial integrity. OXPHOS-Cl-GM-ADP plus pyruvate measured $\mathrm{Cl}$ flux, and OXPHOS-Cl, $\mathrm{Cll}$-represents the addition of succinate to maximize Complexes I and II flux in OXPHOS. Cl, Cll-Leak-Atractyloside (Atr) inhibits OXPHOS and forces leak respiration with $\mathrm{Cl}$ and $\mathrm{ClI}$, while ETS-CI, $\mathrm{Cll}$-results from sequential titration of FCCP to uncouple the electron transport system from OXPHOS. Respiration states used in this study are indicated (vertical text bolded), Bg, background; Leak-GM, leak respiration with GM; OXPHOS-Cl, oxidative phosphorylation supported by Complex I (Cl) substrates; OXPHOS-Cl, Cll, OXPHOS supported by $\mathrm{Cl}$ and Complex II (CII) substrates; ETS, electron transport system (ETS) flux supported by $\mathrm{Cl}$ and $\mathrm{CII}$; ETS-II, ETS supported by $\mathrm{Cll}$; and $\mathrm{CCO}$, cytochrome C oxidase. ETS-Cll—was measured by the addition of rotenone (Rot). Lastly CCO results from the inhibition of Complex III with antimycin-a (Ama), followed by the addition of TMPD-Asc, N, N, N', N'-tetramethyl-p-phenylendiamine + ascorbate; Cat, catalase (concentrations are outlined in the methods); GM, glutamate + malate; ADP, adenosine diphosphate; cyt-c, cytochrome-c; Succ, succinate; atr, atrylactoside; rot, rotenone; Ama, Antimycin A. boiled for $5 \mathrm{~min}$, and separated by SDS-polyacrylamide gel electrophoresis (SDS-PAGE). Protein was transferred to PVDF membranes using a semi-dry Trans-Blot ${ }^{\circledR}$ Turbo $^{\mathrm{TM}}$ Transfer System (Biorad) with ready-to-use Trans-Blot ${ }^{\circledR}$ Turbo $^{\mathrm{TM}}$ Mini PVDF Transfer Packs (Biorad). Membranes were blocked in 5\% BSA/Tris Buffer Saline/0.1\% Tween 20 (TBST) for $2 \mathrm{~h}$ at room temperature, followed by incubation overnight with gentle agitation at $4^{\circ} \mathrm{C}$ with an anti-PGC-1 Antibody (Millipore, AB3242) or an optimized premixed cocktail antibody (Abcam, ab110411, MitoProfile ${ }^{\circledR}$ Total OXPHOS Human) for oxidative phosphorylation proteins (Abcam, Science Park Cambridge, UK) targeting CI subunit NDUFB8 (ab110242/MS105), CII subunit $30 \mathrm{kDa}$ (ab14714/MS203), Complex III subunit Core 2 (ab14745/MS304), Complex IV subunit (CCO) II (ab110258/MS405), and ATP synthase subunit alpha (ab14748/MS507). Membranes were washed for $30 \mathrm{~min}$ with TBST and probed with goat anti-rabbit (for PGC-1 $\alpha$ ) or anti-mouse (for MitoProfile) IgG peroxidase conjugated secondary antibodies (Thermo Scientific Pierce) for $1 \mathrm{~h}$ at room temperature. Membranes were washed for $30 \mathrm{~min}$ in TBST and protein bands were visualized using ECL Prime Western Blotting Detection Reagent (GE healthcare Amersham), and signals quantified using ImageQuant LAS 4000 (FUJI film). Densitometry analysis employed Kodak Molecular Imaging
Software (Version 4.0.5, (1994-2005 Eastman Kodak Company). Equal protein loading was verified by stripping and reprobing membranes with anti-GAPDH (6C5) (Mouse Monoclonal IgG1, Abcam: ab8245).

\section{STATISTICAL ANALYSES}

Paired sampled $t$-tests were used to test between pre- and post-training measures. Diagnostic plots of residuals and fitted values were checked to ensure homogeneity of variance (a key assumption for $t$-tests). Consequently, all data were $\log 10$-transformed and analyses were conducted on these transformed scales. Statistical significance was set at $p \leq 0.05$ and all data are presented as mean \pm SEM unless otherwise stated. Note that the statistical significance reported in the figures is based on analysis of the transformed data but the reported means \pm SEM are on the original (untransformed) scale.

\section{RESULTS}

\section{PERFORMANCE MEASURES}

Following 2 weeks of HIT, peak power and time to fatigue increased by 22 and $33 \%$, respectively $(P<0.01)$. There was no significant increase in relative $\mathrm{VO}_{2}$ peak following training. All performance data are reported in Table $\mathbf{1}$. 


\section{CITRATE SYNTHASE ACTIVITY}

In post-HIT samples, CS activity increased by $43 \%(P<0.05$, Table 1) when compared to baseline pre-training levels.

\section{MITOCHONDRIAL FUNCTION}

A representative trace of the respiration substrate, inhibitor and uncoupler titration protocol is shown in Figure 1. Respiratory

Table 1 | Performance and mitochondrial data pre- and post-HIT.

\begin{tabular}{|c|c|c|c|}
\hline & Pre-HIT & Post-HIT & $P$-value \\
\hline \multicolumn{4}{|l|}{ A. PERFORMANCE } \\
\hline $\mathrm{VO}_{2}$ peak $\left(\mathrm{ml} \cdot \mathrm{kg}^{-1} \cdot \mathrm{min}^{-1}\right)$ & $45.7 \pm 2.1$ & $50.8 \pm 1.0$ & 0.124 \\
\hline Peak power $(\mathrm{W})$ & $151.2 \pm 6.4$ & $184.5 \pm 9.1$ & 0.002 \\
\hline Time to fatigue (s) & $809.3 \pm 51.3$ & $1075.7 \pm 72.8$ & 0.002 \\
\hline \multicolumn{4}{|l|}{ B. MITOCHONDRIAL } \\
\hline RCR-1 & $11.2 \pm 1.3$ & $16.3 \pm 3.4$ & 0.459 \\
\hline RCR-2 & $3.5 \pm 0.1$ & $4.1 \pm 0.3$ & 0.161 \\
\hline$\% \mathrm{Cl}$ of ETS & $47.4 \pm 3.3$ & $50.9 \pm 2.4$ & 0.541 \\
\hline CCO/ETS & $2.0 \pm 0.1$ & $2.2 \pm 0.1$ & 0.120 \\
\hline $\begin{array}{l}\text { Citrate synthase (CS) } \\
\left(\mu \mathrm{mol} \cdot \mathrm{min}^{-1} \cdot \mathrm{mg}^{-1} \text { wet wgt }\right)\end{array}$ & $0.50 \pm 0.1$ & $0.88 \pm 0.1$ & 0.038 \\
\hline
\end{tabular}

Effects of HIT on performance measures, control ratios, fluxes and citrate synthase activity pre- and post-HIT. The RCR-1 represents the ratio of oxidative phosphorylation (OXPHOS) derived oxygen flux relative to leak respiration supported by complex I (CI) substrates (see Figure 1). The RCR-2 represents the ratio of OXPHOS and leak respiration fluxes supported by $\mathrm{Cl}$ and Complex II substrates. The addition of rotenone permitted an estimate of the percentage contribution of $\mathrm{Cl}$ to the chemically uncoupled electron transport system oxygen flux (ETS). The ratio of cytochrome $c$ oxidase (CCO) relative to the ETS (CCO/ETS) provided a measure of the flux through CCO relative to the ETS. Data are presented as mean \pm SEM $(n=8)$. fluxes in response to HIT are shown in Figure 2. The addition of exogenous cytochrome-c provided no evidence of compromised mitochondrial membrane integrity. The corresponding respiratory flux (CYT-C) increased by $21 \%$ post-HIT when compared to baseline pre-HIT levels $(P<0.05)$.

The effect of pyruvate (Pyr) was used to maximize CI mediated flux (OXPHOS-CI), followed by the addition of the Complex II (CII) substrate succinate (S) to maximize combined CI and CII flux (OXPHOS-CI, CII). While the respiratory flux for OXPHOS CI increased with HIT, this increase did not reach statistical significance $(P=0.07)$. Addition of succinate led to a $21 \%$ increase in respiratory flux (OXPHOS CI, II) in permeabilized fibers following HIT $(P<0.001)$. However, when respiration rates were expressed per unit of CS, no significant differences were apparent in CYT-C or OXPHOS-CI, II (data not shown).

Following this, the phosphorylation system was inhibited by addition of atractyloside (Atr) and the electron transport system (ETS) was then uncoupled from the inhibited phosphorylation system by FCCP titrations. Finally, CI (ETS CII), CII and III activity were selectively and sequentially inhibited by the addition of rotenone (Rot), malonate and antimycin-a (AMA). Lastly, the activity of CCO was measured by addition of the electron donor couple $\mathrm{N}, \mathrm{N}, \mathrm{N}^{\prime}, \mathrm{N}^{\prime}$-tetramethyl-p-phenylendiamine (TMPD) and ascorbate. No difference was noted between pre- and posttraining for any of these respiration fluxes. RCR-1, RCR-2, \% CI of ETS and COO/ETS remained unchanged following training (Table 1).

\section{IMMUNOBLOT ANALYSES}

Immunoblot blot analysis of PGC- $1 \alpha$ protein expression showed no significant effect of HIT (Figure 3A). As such, representative blots showing PGC-1 $\alpha$ are displayed (Figure 3B). Similarly, none

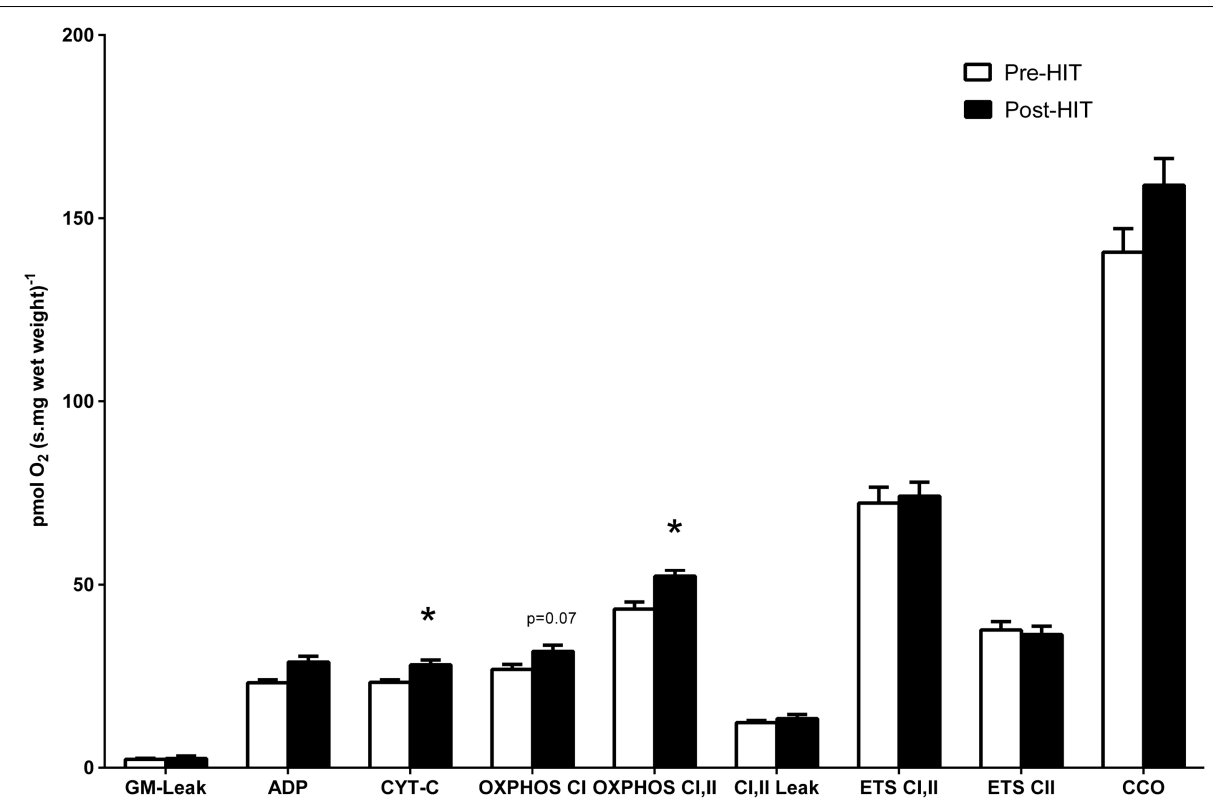

FIGURE 2 | In response to HIT, respiratory flux showed elevation of CYT-C and OXPHOS Cl, II. *Denotes significance $(P<0.05)$, error bars represent SEM, $n=8$. Note that the statistical significance reported in the figures is based on analysis of the transformed data but the reported means \pm SEM are on the original (untransformed) scale. 


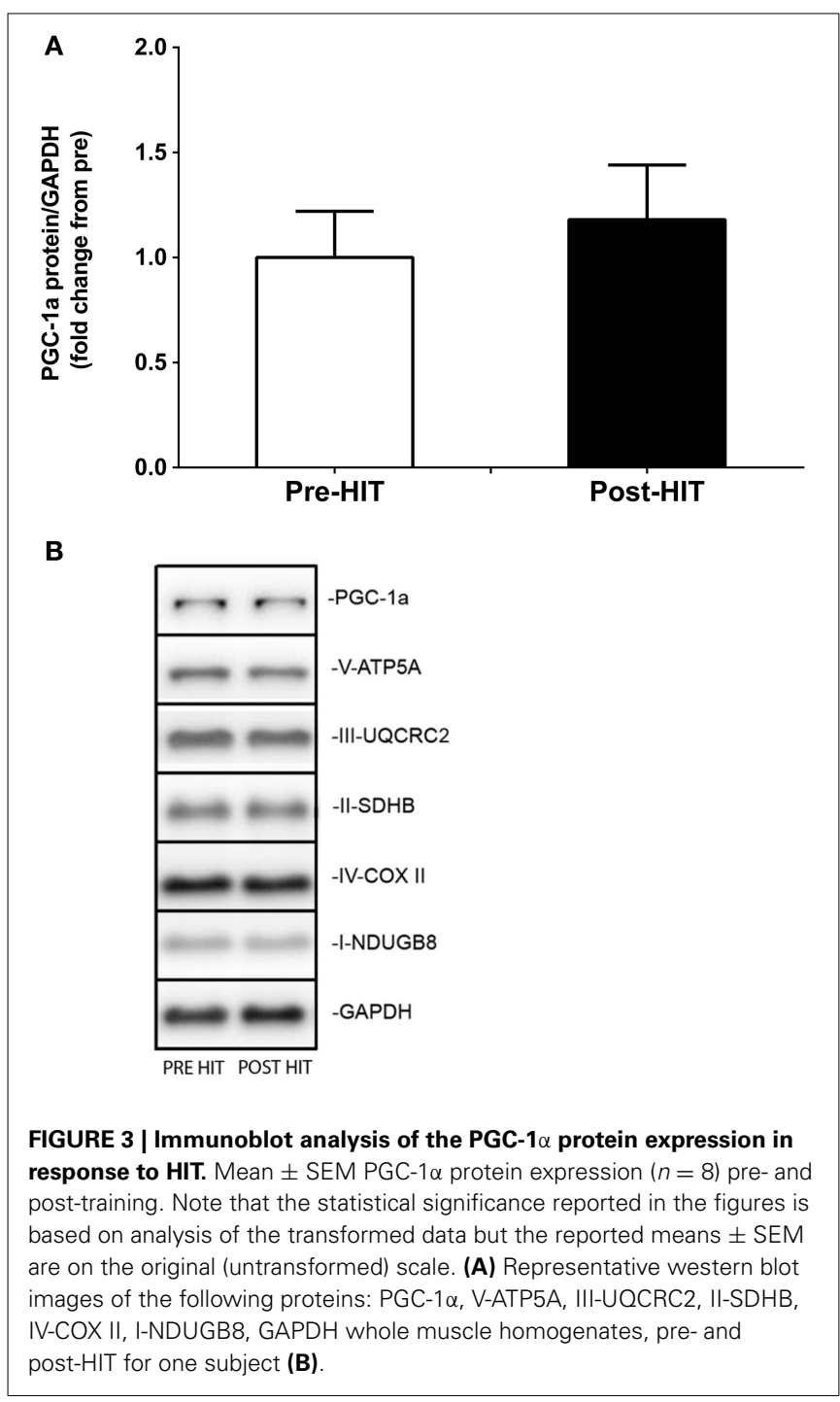

of the mitochondrial specific markers of OXPHOS or ETS measured (PGC-1 $\alpha$, V-ATP5A, III-UQCRC2, II-SDHB, IV-COX II, I-NDUGB8, GAPDH) could resolve differences due to HIT (Figure 3B).

\section{DISCUSSION}

This study is the first to simultaneously evaluate the effects of HIT on mitochondrial respiration and expression of mitochondrialassociated proteins. We showed that only 2 weeks of lowvolume HIT improved exercise performance and increased mitochondrial respiration flux capacities. However, we detected no increases in protein expression of PGC- $1 \alpha$ or OXPHOS or ETS mitochondrial markers. Increased leg muscle power was mirrored by elevated OXPHOS supported combined complex I and II electron inputs (OXPHOS-CI, CII), and this increase matched increases in citrate synthase enzyme activities, a marker of mitochondrial content (Larsen et al., 2012). Our findings indicate that low-volume HIT improves muscle oxidative capacity, mostly through an increase in mitochondrial mass/volume.
The respective improvements of 18 and 25\% for peak power and time to fatigue observed in this study are comparable to previous work involving similar training protocols (Little et al., 2011) or overall training intensities and durations (Rodas et al., 2000). While no significant increase in $\mathrm{VO}_{2}$ peak following 2 weeks of HIT has been reported before (Burgomaster et al., 2005, 2006), minor increases have also been observed (Jacobs et al., 2013b). HIT protocols of a longer duration ( 6 weeks) do however increase $\mathrm{VO}_{2}$ peak to the same extent as traditional endurance training (Burgomaster et al., 2008).

HIT significantly increased maximal OXPHOS flux supported by Complex I and Complex II substrates (OXPHOS-CI, CII), while a trend was apparent for OXPHOS supported by Complex I (CI) substrates only. Others have reported improvements in in vivo oxidative capacity following six sessions of HIT using phosphorous magnetic resonance spectroscopy (Forbes et al., 2008; Larsen et al., 2013). Maximal OXPHOS with CI and CII is consistent with others findings employing the permeabilized fiber method with a short HIT protocol (Jacobs et al., 2013b). Jacobs et al. (2013b) also reported an increase in OXPHOS and ETS flux with multiple electron inputs (including lipids via electron transferring flavoprotein, ETF), but not with just the complex II substrate succinate. While the lack of significance in ETS states may reflect the relatively small sample size of our study $(n=8)$ and/or the lower overall training duration across the 2 weeks period, the activation of both CI and CII appears to better reveal muscle mitochondrial adaptation to HIT.

Compared to pre-HIT values, we observed a $43 \%$ increase in CS activity, an elevation that is greater than in other studies with similar effort and duration (Rodas et al., 2000; Little et al., 2010). CS activity is a quantitative indicator of oxidative capacity and is used to normalize global measures of muscle bioenergetic capacity (Larsen et al., 2012). On normalizing OXPHOS-CI, CII capacity to citrate synthase activities no differences were apparent between pre- and post-training respiratory states. We also report no significant improvement in the RCR-1, RCR-2, \% CI ETS, and the ratio of cytochrome $c$ oxidase (CCO) relative to the ETS (CCO/ETS) in muscle fibers. Increases in RCRs theoretically indicate greater ATP yields per oxygen molecule and therefore greater OXPHOS efficiency (Pesta and Gnaiger, 2012), as reported in active/athletic vs. sedentary individuals (Zoll et al., 2004; Coen et al., 2013). Similarly, changes in CCO/ETS indicate alterations in pathway flux control and efficiencies (Pesta and Gnaiger, 2012). Our results suggest that alterations to muscle oxidative capacity are mostly enhanced through quantitative rather than qualitative changes in mitochondria. Still an explanation is required for the more substantive changes in time to fatigue and peak power output relative to the lesser increases in mitochondrial flux ( 10-15\%). Mitochondria need not operate at full capacity, as respiration of permeabilised vastus lateralis muscle fibres is greater in vitro than the apparent respiration rates of thigh muscle measured in vivo. However, these improvements may also result from increased non-bicarbonate muscle $\mathrm{pH}$ buffering capacities (Edge et al., 2006; Gibala et al., 2006b), a parameter not measured in this study.

In contrast to changes in mitochondrial function, there was no change in the protein expression of PGC- $1 \alpha$ or of mitochondrial markers of OXPHOS or ETS. Endurance (Russell et al., 
2003) and HIT protocols (Gibala et al., 2009) have reported increases in PGC-1 $\alpha$ mRNA and protein contents. However, discrepancies between alterations in the mitochondrial function and associated changes in protein expression have previously been noted (Viganò et al., 2008; Jacobs et al., 2013a). Total PGC$1 \alpha$ protein content may not reflect PCG-1 $\alpha$ activation (Wright et al., 2007b). On activation PGC- $1 \alpha$ translocates to the nucleus (Rim et al., 2004; Cowell et al., 2007; Sano et al., 2007; Wright et al., 2007b) and does so in stimulation by HIT (Little et al., 2010). Translocation was not testable in muscle homogenates in our study. However, prolonged (6 weeks) HIT increased the whole muscle protein content of PGC- $1 \alpha$ by $100 \%$ in young healthy individuals (Burgomaster et al., 2008). Thus, mitochondrial capacity appears to improve through expansion prior to measurable changes in PGC-1 $\alpha$ expression with limited exposure to HIT.

HIT also did not induce measurable changes in the protein expression levels of the respiratory complex components CI subunit NDUFB8, CII subunit $30 \mathrm{kDa}$, CIII subunit Core 2, CIV subunit (CCO) II and ATP synthase subunit alpha. CS is a validated marker of mitochondrial biogenesis (Larsen et al., 2012). However, it was also suggested that complex II and complex IV protein content are alternative suitable markers of mitochondrial content (Larsen et al., 2012). In the present study, despite an increase in CS activity, we observed no change in the levels of CII and CCO proteins. This may be indicative of intrinsic alterations of mitochondrial function. Qualitative changes in the OXPHOS process, or regulators of mitochondrial respirational flux (e.g., NOS and NO) might partly account for the observed increase in respiration rates. This may in turn explain the lack of change in the expression levels of respiratory complex components.

In conclusion, the current study demonstrates that skeletal muscle mitochondrial function, but not mitochondrialassociated protein expression, is modestly altered following only 2 weeks of HIT in young untrained males. It indicates that HIT provides a time-efficient training strategy for improving skeletal muscle mitochondrial function.

\section{ACKNOWLEDGMENTS}

The authors would like to acknowledge Dr. Johann (Hans) Edge who was tragically killed while cycling home in 2010. Hans was a devoted husband and father of three, and an inspirer of young minds. Without his leadership, insight, and drive this study would not have been possible. We thank all participants who took part and those who provided support for this project including Chris Hedges, Kurt Olsen, and Briar Rudsits. This project was partially supported by the Physiological Society Vacation Studentship.

\section{REFERENCES}

Baar, K., Song, Z., Semenkovich, C. F., Jones, T. E., Han, D.-H., Nolte, L. A., et al. (2003). Skeletal muscle overexpression of nuclear respiratory factor 1 increases glucose transport capacity. FASEB J. 17, 1666-1673. doi: 10.1096/fj.03-0049com

Babraj, J. A., Vollaard, N. B., Keast, C., Guppy, F. M., Cottrell, G., and Timmons, J. A. (2009). Extremely short duration high intensity interval training substantially improves insulin action in young healthy males. BMC Endocr. Disord. 9:3. doi: 10.1186/1472-6823-9-3

Bartlett, J. D., Close, G. L., Maclaren, D. P., Gregson, W., Drust, B., and Morton, J. P. (2011). High-intensity interval running is perceived to be more enjoyable than moderate-intensity continuous exercise: implications for exercise adherence. J. Sports sci. 29, 547-553. doi: 10.1080/02640414.2010.545427

Boushel, R., Gnaiger, E., Calbet, J. A., Gonzalez-Alonso, J., Wright-Paradis, C., Saltin, B. et al. (2011). Muscle mitochondrial capacity exceeds maximal oxygen delivery in humans. Mitochondrion 11, 303-307. doi: 10.1016/j.mito.2010.12.006

Bruce, C., Kriketos, A., Cooney, G., and Hawley, J. (2004). Disassociation of muscle triglyceride content and insulin sensitivity after exercise training in patients with Type 2 diabetes. Diabetologia 47, 23-30. doi: 10.1007/s00125-0031265-7

Burgomaster, K. A., Cermak, N. M., Phillips, S. M., Benton, C. R., Bonen, A., and Gibala, M. J. (2007). Divergent response of metabolite transport proteins in human skeletal muscle after sprint interval training and detraining. Am. J. Physiol. Regul. Integr. Comp. Physiol. 292, R1970-R1976. doi: 10.1152/ajpregu.00503.2006

Burgomaster, K. A., Heigenhauser, G. J., and Gibala, M. J. (2006). Effect of short-term sprint interval training on human skeletal muscle carbohydrate metabolism during exercise and time-trial performance. J. Appl. Physiol. 100, 2041-2047. doi: 10.1152/japplphysiol.01220.2005

Burgomaster, K. A., Howarth, K. R., Phillips, S. M., Rakobowchuk, M., MacDonald, M. J., McGee, S. L., et al. (2008). Similar metabolic adaptations during exercise after low volume sprint interval and traditional endurance training in humans. J. Physiol. 586, 151-160. doi: 10.1113/jphysiol.2007.142109

Burgomaster, K. A., Hughes, S. C., Heigenhauser, G. J., Bradwell, S. N., and Gibala, M. J. (2005). Six sessions of sprint interval training increases muscle oxidative potential and cycle endurance capacity in humans. J. Appl. Physiol. 98, 1985-1990. doi: 10.1152/japplphysiol.01095.2004

Cartoni, R., Léger, B., Hock, M. B., Praz, M., Crettenand, A., Pich, S., et al. (2005). Mitofusins $1 / 2$ and $\operatorname{ERR} \alpha$ expression are increased in human skeletal muscle after physical exercise. J. Physiol. 567, 349-358. doi: 10.1113/jphysiol.2005.092031

Coen, P. M., Jubrias, S. A., Distefano, G., Amati, F., Mackey, D. C., Glynn, N. W., et al. (2013). Skeletal muscle mitochondrial energetics are associated with maximal aerobic capacity and walking speed in older adults. J. Gerontol. A Biol. Sci. Med. Sci. 68, 447-455. doi: 10.1093/gerona/gls196

Cowell, R. M., Blake, K. R., and Russell, J. W. (2007). Localization of the transcriptional coactivator PGC- $1 \alpha$ to GABAergic neurons during maturation of the rat brain. J. Comp. Neurol. 502, 1-18. doi: 10.1002/cne.21211

Daussin, F. N., Zoll, J., Ponsot, E., Dufour, S. P., Doutreleau, S., Lonsdorfer, E., et al. (2008). Training at high exercise intensity promotes qualitative adaptations of mitochondrial function in human skeletal muscle. J. Appl. Physiol. 104, 1436-1441. doi: 10.1152/japplphysiol.01135.2007

Edge, J., Bishop, D., and Goodman, C. (2006). Effects of chronic $\mathrm{NaHCO}_{3}$ ingestion during interval training on changes to muscle buffer capacity, metabolism, and short-term endurance performance. J. Appl. Physiol. 101, 918-925. doi: 10.1152/japplphysiol.01534.2005

Finck, B. N., and Kelly, D. P. (2006). PGC-1 coactivators: inducible regulators of energy metabolism in health and disease. J. Clin. Invest. 116, 615-622. doi: 10.1172/JCI27794

Forbes, S. C., Slade, J. M., and Meyer, R. A. (2008). Short-term high-intensity interval training improves phosphocreatine recovery kinetics following moderateintensity exercise in humans. Appl. Physiol. Nutr. Metab. 33, 1124-1131. doi: 10.1139/H08-099

Gibala, M. J., Little, J. P., Macdonald, M. J., and Hawley, J. A. (2012). Physiological adaptations to low-volume, high-intensity interval training in health and disease. J. Physiol. 590, 1077-1084. doi: 10.1113/jphysiol.2011.224725

Gibala, M. J., Little, J. P., van Essen, M., Wilkin, G. P., Burgomaster, K. A., Safdar, A., et al. (2006a). Short-term sprint interval versus traditional endurance training: similar initial adaptations in human skeletal muscle and exercise performance. J. Physiol. 575, 901-911. doi: 10.1113/jphysiol.2006. 112094

Gibala, M. J., Little, J. P., van Essen, M., Wilkin, G. P., Burgomaster, K. A., Safdar, A., et al. (2006b). Short-term sprint interval versus traditional endurance training: similar initial adaptations in human skeletal muscle and exercise performance. J. Physiol. 575, 901-911. doi: 10.1113/jphysiol.2006.112094

Gibala, M. J., Little, J. P., van Essen, M., Wilkin, G. P., Burgomaster, K. A., Safdar, A., et al. (2006c). Short-term sprint interval versus traditional endurance training: similar initial adaptations in human skeletal muscle and exercise performance. J. Physiol. 575, 901-911. doi: 10.1113/jphysiol.2006.112094 
Gibala, M. J., and McGee, S. L. (2008). Metabolic adaptations to short-term highintensity interval training: a little pain for a lot of gain? Exerc. Sport Sci. Rev. 36, 58-63. doi: 10.1097/JES.0b013e318168eclf

Gibala, M. J., McGee, S. L., Garnham, A. P., Howlett, K. F., Snow, R. J., and Hargreaves, M. (2009). Brief intense interval exercise activates AMPK and p38 MAPK signaling and increases the expression of PGC-1 $\alpha$ in human skeletal muscle. J. Appl. Physiol. 106, 929-934. doi: 10.1152/japplphysiol.90880.2008

Handschin, C., and Spiegelman, B. M. (2006). Peroxisome proliferator-activated receptor $\gamma$ coactivator 1 coactivators, energy homeostasis, and metabolism. Endocr. Rev. 27, 728-735. doi: 10.1210/er.2006-0037

Holloszy, J. O. (1967). Biochemical adaptations in muscle effects of exercise on mitochondrial oxygen uptake and respiratory enzyme activity in skeletal muscle. J. Biol. Chem. 242, 2278-2282.

Hood, M. S., Little, J. P., Tarnopolsky, M. A., Myslik, F., and Gibala, M. J. (2011). Low-volume interval training improves muscle oxidative capacity in sedentary adults. Med. Sci. Sports Exerc. 43, 1849-1856. doi: 10.1249/MSS.0b013e3182199834

Howatson, G., Zult, T., Farthing, J. P., Zijdewind, I., and Hortobágyi, T. (2013). Mirror training to augment cross-education during resistance training: a hypothesis. Front. Hum. Neurosci. 7:396. doi: 10.3389/fnhum.2013.00396

Irrcher, I., Ljubicic, V., and Hood, D. A. (2009). Interactions between ROS and AMP kinase activity in the regulation of PGC- $1 \alpha$ transcription in skeletal muscle cells. Am. J. Physiol. Cell Physiol. 296, C116-C123. doi: 10.1152/ajpcell.00267.2007

Jacobs, R. A., Boushel, R., Wright-Paradis, C., Calbet, J. A., Robach, P., Gnaiger, E., et al. (2013a). Mitochondrial function in human skeletal muscle following high-altitude exposure. Exp. Physiol. 98, 245-255. doi: 10.1113/expphysiol.2012.066092

Jacobs, R. A., Flück, D., Bonne, T. C., Bürgi, S., Christensen, P. M., Toigo, M., et al. (2013b). Improvements in exercise performance with high-intensity interval training coincide with an increase in skeletal muscle mitochondrial content and function. J. Appl. Physiol. 115, 785-793. doi: 10.1152/japplphysiol.00445.2013

Larsen, R. G., Befroy, D. E., and Kent-Braun, J. A. (2013). High-intensity interval training increases in vivo oxidative capacity with no effect on Pi?ATP rate in resting human muscle. Am. J. Physiol. Regul. Integr. Comp. Physiol. 304, R333-R342. doi: 10.1152/ajpregu.00409.2012

Larsen, S., Nielsen, J., Hansen, C. N., Nielsen, L. B., Wibrand, F., Stride, N., et al. (2012). Biomarkers of mitochondrial content in skeletal muscle of healthy young human subjects. J. Physiol. 590, 3349-3360. doi: 10.1113/jphysiol.2012.230185

Little, J. P., Safdar, A., Bishop, D., Tarnopolsky, M. A., and Gibala, M. J. (2011). An acute bout of high-intensity interval training increases the nuclear abundance of PGC- $1 \alpha$ and activates mitochondrial biogenesis in human skeletal muscle. Am. J. Physiol. Regul. Integr. Comp. Physiol. 300, R1303-R1310. doi: 10.1152/ajpregu.00538.2010

Little, J. P., Safdar, A., Wilkin, G. P., Tarnopolsky, M. A., and Gibala, M. J. (2010). A practical model of low-volume high-intensity interval training induces mitochondrial biogenesis in human skeletal muscle: potential mechanisms. J. Physiol. 588, 1011-1022. doi: 10.1113/jphysiol.2009.181743

Magnus, C. R., Arnold, C. M., Johnston, G., Dal-Bello Haas, V., Basran, J., Krentz, J. R., et al. (2013). Cross-education for improving strength and mobility after distal radius fractures: a randomized controlled trial. Arch. Phys. Med. Rehabil. 94, 1247-1255. doi: 10.1016/j.apmr.2013.03.005

McConell, G. K., Burge, C. M., Skinner, S. L., and Hargraves, M. (1997). Infuence of ingested fuid volume on physiological responses during prolonged exercise. Acta Physiol. Scand. 160, 149-156. doi: 10.1046/j.1365-201X.1997.00139.x

Parra, J., Cadefau, J., Rodas, G., Amigo, N., and Cusso, R. (2000). The distribution of rest periods affects performance and adaptations of energy metabolism induced by high-intensity training in human muscle. Acta Physiol. Scand. 169, 157-166. doi: 10.1046/j.1365-201x.2000.00730.x

Pearce, A., Hendy, A., Bowen, W., and Kidgell, D. (2013). Corticospinal adaptations and strength maintenance in the immobilized arm following 3 weeks unilateral strength training. Scand. J. Med. Sci. Sports 23, 740-748. doi: 10.1111/j.16000838.2012.01453.x

Pesta, D., and Gnaiger, E. (2012). High-resolution respirometry: OXPHOS protocols for human cells and permeabilized fibers from small biopsies of human muscle. Methods Mol. Biol. 810, 25-58. doi: 10.1007/978-1-61779-382-0_3

Picard, M., Taivassalo, T., Gouspillou, G., and Hepple, R. T. (2011). Mitochondria: isolation, structure and function. J. Physiol. 589, 4413-4421. doi: 10.1113/jphysiol.2011.212712
Rakobowchuk, M., Tanguay, S., Burgomaster, K. A., Howarth, K. R., Gibala, M. J., and Macdonald, M. J. (2008). Sprint interval and traditional endurance training induce similar improvements in peripheral arterial stiffness and flow-mediated dilation in healthy humans. Am. J. Physiol. Regul. Integr. Comp. Physiol. 295, R236-R242. doi: 10.1152/ajpregu.00069.2008

Rim, J. S., Xue, B., Gawronska-Kozak, B., and Kozak, L. P. (2004). Sequestration of thermogenic transcription factors in the cytoplasm during development of brown adipose tissue. J. Biol. Chem. 279, 25916-25926. doi: 10.1074/jbc.M402102200

Rodas, G., Ventura, J. L., Cadefau, J. A., Cusso, R., and Parra, J. (2000). A short training programme for the rapid improvement of both aerobic and anaerobic metabolism. Eur. J. Appl. Physiol. 82, 480-486. doi: 10.1007/s004210000223

Russell, A. P., Feilchenfeldt, J., Schreiber, S., Praz, M., Crettenand, A., Gobelet, C., et al. (2003). Endurance training in humans leads to fiber type-specific increases in levels of peroxisome proliferator-activated receptor- $\gamma$ coactivator1 and peroxisome proliferator-activated receptor- $\alpha$ in skeletal muscle. Diabetes 52, 2874-2881. doi: 10.2337/diabetes.52.12.2874

Russell, A. P., Hesselink, M. K., Lo, S. K., and Schrauwen, P. (2005). Regulation of metabolic transcriptional co-activators and transcription factors with acute exercise. FASEB J. 19, 986-988. doi: 10.1096/fj.04-3168fje

Sano, M., Tokudome, S., Shimizu, N., Yoshikawa, N., Ogawa, C., Shirakawa, K., et al. (2007). Intramolecular control of protein stability, subnuclear compartmentalization, and coactivator function of peroxisome proliferatoractivated receptor $\gamma$ coactivator $1 \alpha$. J. Biol. Chem. 282, 25970-25980. doi: 10.1074/jbc.M703634200

Tjonna, A. E., Lee, S. J., Rognmo, O., Stolen, T. O., Bye, A., Haram, P. M., et al. (2008). Aerobic interval training versus continuous moderate exercise as a treatment for the metabolic syndrome: a pilot study. Circulation 118, 346-354. doi: 10.1161/CIRCULATIONAHA.108.772822

Viganò, A., Ripamonti, M., De Palma, S., Capitanio, D., Vasso, M., Wait, R., et al. (2008). Proteins modulation in human skeletal muscle in the early phase of adaptation to hypobaric hypoxia. Proteomics 8, 4668-4679. doi: 10.1002/pmic. 200800232

Wright, D. C., Geiger, P. C., Han, D.-H., Jones, T. E., and Holloszy, J. O. (2007a). Calcium induces increases in peroxisome proliferator-activated receptor $\gamma$ coactivator- $1 \alpha$ and mitochondrial biogenesis by a pathway leading to p38 mitogen-activated protein kinase activation. J. Biol. Chem. 282, 18793-18799. doi: 10.1074/jbc.M611252200

Wright, D. C., Han, D.-H., Garcia-Roves, P. M., Geiger, P. C., Jones, T. E., and Holloszy, J. O. (2007b). Exercise-induced mitochondrial biogenesis begins before the increase in muscle PGC-1 $\alpha$ expression. J. Biol. Chem. 282, 194-199. doi: 10.1074/jbc.M606116200

Wu, Z., Puigserver, P., Andersson, U., Zhang, C., Adelmant, G., Mootha, V., et al. (1999). Mechanisms controlling mitochondrial biogenesis and respiration through the thermogenic coactivator PGC-1. Cell 98, 115-124. doi: 10.1016/S0092-8674(00)80611-X

Zoll, J., Sanchez, H., N'Guessan, B., Ribera, F., Lampert, E., Bigard, X., et al. (2004). Physical activity changes the regulation of mitochondrial respiration in human skeletal muscle. J. Physiol. 543, 191-200. doi: 10.1113/jphysiol.2002.019661

Conflict of Interest Statement: The authors declare that the research was conducted in the absence of any commercial or financial relationships that could be construed as a potential conflict of interest.

Received: 30 November 2014; accepted: 05 February 2015; published online: 24 February 2015.

Citation: Vincent G, Lamon S, Gant N, Vincent PJ, MacDonald JR, Markworth JF, Edge JA and Hickey AJR (2015) Changes in mitochondrial function and mitochondria associated protein expression in response to 2-weeks of high intensity interval training. Front. Physiol. 6:51. doi: 10.3389/fphys.2015.00051

This article was submitted to Striated Muscle Physiology, a section of the journal Frontiers in Physiology.

Copyright (c) 2015 Vincent, Lamon, Gant, Vincent, MacDonald, Markworth, Edge and Hickey. This is an open-access article distributed under the terms of the Creative Commons Attribution License (CC BY). The use, distribution or reproduction in other forums is permitted, provided the original author(s) or licensor are credited and that the original publication in this journal is cited, in accordance with accepted academic practice. No use, distribution or reproduction is permitted which does not comply with these terms. 